\title{
Nineteen-year follow-up of a patient with severe glutathione synthetase deficiency
}

\author{
Paldeep S Atwal ${ }^{1,2}$, Casey R Medina ${ }^{1}$, Lindsay C Burrage ${ }^{1}$ and V Reid Sutton ${ }^{1}$ \\ Glutathione synthetase deficiency is a rare autosomal recessive disorder resulting in low levels of glutathione and an increased \\ susceptibility to oxidative stress. Patients with glutathione synthetase deficiency typically present in the neonatal period with \\ hemolytic anemia, metabolic acidosis and neurological impairment. Lifelong treatment with antioxidants has been recommended \\ in an attempt to prevent morbidity and mortality associated with the disorder. Here, we present a 19-year-old female who was \\ diagnosed with glutathione synthetase deficiency shortly after birth and who has been closely followed in our metabolic clinic. \\ Despite an initial severe presentation, she has had normal intellectual development and few complications of her disorder with a \\ treatment regimen that includes polycitra (citric acid, potassium citrate and sodium citrate), vitamin $\mathrm{C}$, vitamin $\mathrm{E}$ and selenium. \\ Journal of Human Genetics (2016) 61, 669-672; doi:10.1038/jhg.2016.20; published online 17 March 2016
}

\section{INTRODUCTION}

Glutathione (GSH) is a ubiquitous tripeptide (L-y-glutamyl-L-cysteinylglycine) known to function in many aspects of cellular activity including protein and DNA synthesis, transport, detoxification of xenobiotics and carcinogens, metabolism, and defense against free radicals and oxidative stress. ${ }^{1}$ It is synthesized in the $\gamma$-glutamyl cycle (Figure 1) by GSH synthetase (GS). GSH is most commonly found in its reduced form with only $1-5 \%$ existing in the oxidized form GSH-disulfide. GSH-disulfide is reduced back to GSH by GSH-disulfide reductase requiring one equivalent of NADPH. ${ }^{2}$ Biallelic, pathogenic variants in the GSS gene result in GS deficiency (GSSD).

GSSD (MIM:266130) is a rare autosomal recessive disorder that has only been described in approximately 70 individuals worldwide. ${ }^{3}$ Erythrocytes have some of the highest concentrations of GSH in the body, and thus, erythrocytes from patients with GSSD are more susceptible to oxidative stress. As a result, hemolytic anemia is a common problem in GSSD. ${ }^{4}$ Depending on their clinical manifestations, patients can be divided into mild, moderate or severe phenotypes. Patients with the mild GSSD present with isolated hemolytic anemia as their only clinical symptom, whereas patients with the moderate form of the disorder present with hemolytic anemia and metabolic acidosis in the neonatal period. In addition to these symptoms, patients with severe disease have neurologic findings including motor disturbances and developmental delay. ${ }^{5}$ Some severely affected patients show an increased susceptibility to bacterial infections, thought to be due to defective granulocyte function. To date, there is only one severely affected individual who was treated with antioxidants from birth and long-term follow-up on that individual is not reported. 5-Oxoprolinuria can also be found in all patients with GSSD due to the accumulation of $\gamma$-glutamylcysteine being hydrolyzed to 5-oxoproline and cysteine by $\gamma$-glutamyl cyclotransferase. This accumulation of 5-oxoproline exceeds the capacity of 5-oxoprolinase leading to high urinary excretion of 5-oxoproline that is detectable on urine organic acid analysis. ${ }^{6}$ In the present report, we describe the long-term follow-up of a case of severe GSSD.

\section{CASE PRESENTATION}

The proband is a female born at 40 weeks gestation by Cesarean section weighing $2.970 \mathrm{~kg}$ (16th centile) with Apgar scores of 8 and 9 at 1 and $5 \mathrm{~min}$, respectively. At approximately $18 \mathrm{~h}$ of life, she was found to be tachypneic with a metabolic acidosis. Arterial blood gas showed $\mathrm{pH}$ of 7.32 (normal $\mathrm{pH}$ 7.35-7.45), $\mathrm{PaCO}_{2} 17 \mathrm{~mm} \mathrm{Hg}$ (normal 35-45 mm Hg), $\mathrm{PaO}_{2} 94 \mathrm{~mm} \mathrm{Hg}$ (normal $80-100 \mathrm{~mm} \mathrm{Hg}$ ) and bicarbonate of $8 \mathrm{mmoll}^{-1}$ (normal 21-32 $\mathrm{mmoll}^{-1}$ ). She was given $5 \mathrm{mg}$ intravenous (IV) sodium bicarbonate and transferred to a tertiary hospital at which time her metabolic acidosis had worsened to $\mathrm{pH} 7.15, \mathrm{PaCO}_{2} 12 \mathrm{~mm} \mathrm{Hg}, \mathrm{PaO}_{2} 94 \mathrm{~mm} \mathrm{Hg}$ and bicarbonate of $4 \mathrm{mmoll}^{-1}$. She was treated with IV fluids and IV sodium bicarbonate of $1.1 \mathrm{meq} \mathrm{kg}^{-1} \mathrm{~h}^{-1}$. Urine organic acid analysis performed at this time revealed high levels of 5-oxoproline, suggesting a defect in GSH metabolism. Once stabilized, she was started on a combination of citric acid, potassium citrate and sodium citrate $\left(\sim 30 \mathrm{meq} \mathrm{kg}^{-1}\right.$ per day) for metabolic acidosis and vitamin C (250 mg per day) and $\mathrm{E}$ (30 international units per day) to help prevent oxidative stress. Her mother was also advised to avoid foods and medications that cause oxidative stress (from a list standardly used for individuals with glucose-6-phosphate dehydrogenase deficiency). She was then discharged home with follow-up in the metabolic clinic.

\footnotetext{
${ }^{1}$ Department of Molecular and Human Genetics, Baylor College of Medicine, Houston, TX, USA and ${ }^{2}$ Department of Clinical Genomics, Center for Individualized Medicine, Mayo Clinic, Jacksonville, FL, USA

Correspondence: Dr VR Sutton, Department of Molecular and Human Genetics, Baylor College of Medicine, One Bayor Plaza, Houston, TX 77030, USA.

E-mail: vrsutton@texaschildrens.org

Received 30 September 2015; revised 17 February 2016; accepted 17 February 2016; published online 17 March 2016
} 


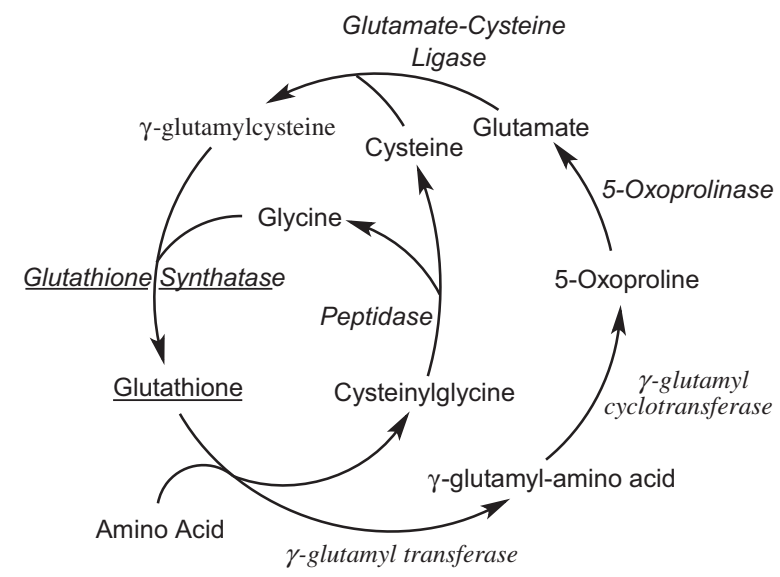

Figure $1 \gamma$-Glutamyl cycle: glutathione is synthesized by glutathione synthetase from glutamine and cysteine in a two-step process involving the enzymes glutathione synthetase and glutamate-cysteine ligase.

Table 1 Results of GS assay

\begin{tabular}{lcc}
\hline & GSH level $\left(\mu \mathrm{Mol} g \mathrm{gb}^{-1}\right)$ & GS activity $\left(I \cup \mathrm{gHb}^{-1}\right)$ \\
\hline Patient & 0.60 & 0.04 \\
Mother & 6.17 & 0.19 \\
Control & 5.44 & 0.45 \\
Normal range & $4.50-8.70$ & $0.24-0.53$ \\
\hline
\end{tabular}

Abbreviations: GS, glutathione synthetase; GSH, glutathione.

At her 2-month follow-up visit at the metabolic clinic, she had remained stable with no intercurrent illnesses or hospitalizations since her discharge. She was tolerating her medications and growth was satisfactory (40th centile for weight, 10th centile for height). Physical exam was normal. Laboratory results showed mild macrocytic anemia with reticulocytosis and hyperkalemia, which became her baseline for most of her life, and a normal bicarbonate of $23 \mathrm{mmoll}^{-1}$. At 6 months, her red blood cell GSH and GS activity levels were analyzed, and she had a marked decrease in both GSH level and GS activity (see Table 1), consistent with the diagnosis of GSSD. Her mother also had a decrease in GS activity, although normal GSH levels, suggesting heterozygosity.

Throughout early childhood, she had multiple episodes of otitis media, which resolved with antibiotics and multiple minor viral respiratory infections. She also developed an episode of tachypnea and acidosis in the setting of 4 days of fever, watery diarrhea, congestion and spitting up. Her serum bicarbonate level was $10 \mathrm{mmoll}^{-1}$, and she was hospitalized. Her acidosis improved with IV fluids and IV bicarbonate. She was hospitalized again at 18 years of age when a viral gastroenteritis caused decreased oral intake and inability to take medications; her serum bicarbonate level at that time was $7 \mathrm{mmoll}^{-1}$ and resolved quickly with hydration and IV bicarbonate. Numerous dental caries were reported, and her dental health improved after a switch to a sugar-free combination of citric acid, potassium citrate and sodium citrate.

At approximately 2 years 3 months of age, there was concern that increased oxidative stress might hinder her developmental progress, so she was started on selenium-one crushed tablet daily. A formal developmental evaluation was performed at approximately 2 years 6 months and was reported as normal. However, by 3 years 9 months, developmental delays were evident. She was unable to copy a circle or ride a tricycle and her language was unintelligible. She was also having difficulties with toilet training. Speech therapy led to significant improvement, and by 4.5 years, her speech had greatly improved and her other developmental concerns resolved.

At her 33 months of age, weight loss and deceleration of head growth were noted, and these findings were attributed to chronic metabolic acidosis (bicarbonate of $15-18 \mathrm{moll}^{-1}$ ), her citric acid, potassium citrate and sodium citrate was adjusted to $\sim 18 \mathrm{meq} \mathrm{kg}^{-1}$ per day and by three years of age, her acidosis and weight gain had improved.

Ophthalmology evaluation for pigmentary retinopathy, a known finding in patients with GSSD, was normal. At the age of 5 years, she underwent a tonsillectomy and adenoidectomy for chronic snoring and mouth breathing without any complications. She was diagnosed with attention deficit disorder at approximately 7 years of age and was successfully treated with guanfacine. At approximately 10 years, she was diagnosed with a seizure disorder and treated with the antiepileptic lamotrigine. However, by the age of 15 years, she had no additional seizures, and her electroencephalogram was normal, so she was successfully weaned off lamotrigine. At the age of 16 years, her psychologist diagnosed her with Asperger syndrome. We are unaware of any association with of Asperger syndrome with GSSD.

Currently, at 19 years, she is doing well. She graduated from mainstream high school and attends community college. She is currently prescribed guanfacine for attention deficit hyperactivity disorder, citric acid, potassium citrate and sodium citrate syrup (11 meq $\mathrm{kg}^{-1}$ per day) for metabolic acidosis, and vitamin $\mathrm{C}$ (14.5 $\mathrm{mg} \mathrm{kg}^{-1}$ per day), vitamin E (150 international units per day) and selenium ( $50 \mathrm{mcg}$ per day) to prevent oxidative stress. Her bicarbonate levels and reticulocyte counts are provided in Figure 2.

\section{DISCUSSION}

GSSD is a metabolic disorder that requires lifelong treatment. Treatment depends on the signs and symptoms of the patient. However, high doses of vitamins $\mathrm{C}$ and $\mathrm{E}$ are recommended in all patients for protection against oxidative stress. ${ }^{3,7}$ Avoidance of foods and drugs known to cause hemolytic crisis in glucose-6-phosphate dehydrogenase deficiency is also important as these same triggers can cause hemolytic crisis in GSSD. ${ }^{8}$ Vitamin $\mathrm{E}$ is also used to prevent granulocyte dysfunction, which could cause recurrent infections. ${ }^{9}, 10$ Patients with moderate and severe phenotypes typically require treatment for metabolic acidosis. In acute crisis, IV bicarbonate is given for immediate correction, and for long-term management, citrate or trometamol are given. Our patient has required very high doses of citric acid, potassium citrate and sodium citrate (ranging from $\sim 30 \mathrm{meq}^{-1}$ per day in early infancy to approximately 10-20 meq kg-1 per day throughout most of her childhood and adolescent years) to maintain a normal serum bicarbonate level (Figure 2). Moreover, her persistently elevated reticulocyte count (Figure 2) reflects ongoing but compensated hemolytic anemia.

Previously, cysteine delivery compounds, specifically N-acetylcysteine, were given because they increase GSH levels in healthy patients. ${ }^{11}$ However, $\mathrm{N}$-acetylcysteine has been found to increase intracellular levels of cysteine, which are already elevated in GSSD ${ }^{6}$ and these high levels of cysteine are known to be neurotoxic. ${ }^{12}$ Selenium was another agent used in our patient to prevent oxidative stress, and to our knowledge, this is the first reported use of selenium in a patient with GSSD. Selenium is found to have strong antioxidant properties through formation of selenoproteins, which are thought to protect against reactive oxygen species. ${ }^{13}$ 


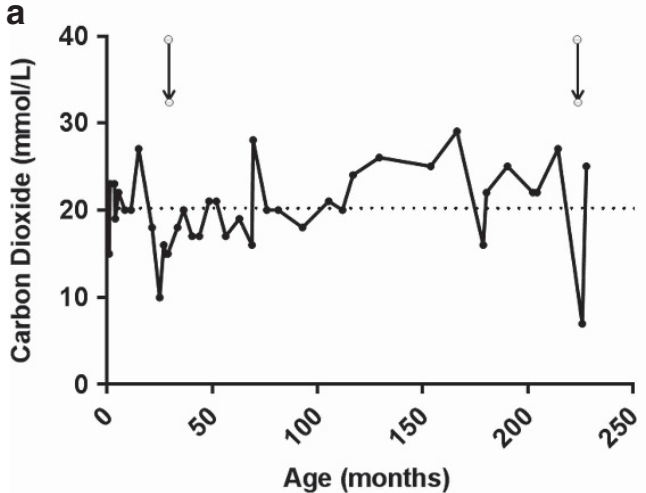

C

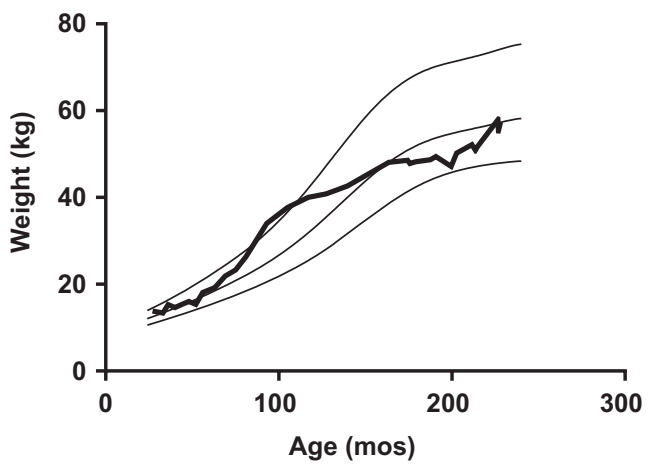

b

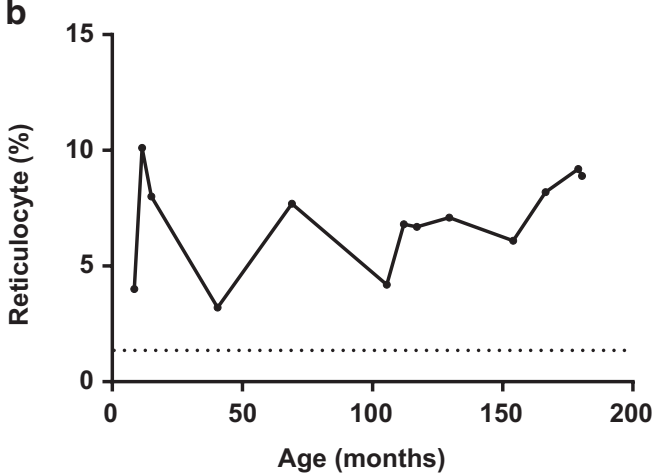

d

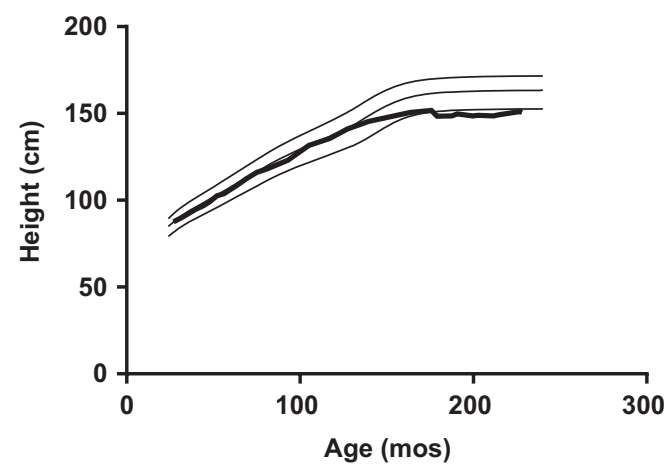

Figure 2 Laboratory results and growth parameters. (a) The proband's bicarbonate levels across her lifespan are plotted by age. Hospitalizations are marked with arrows. To maintain these levels, doses of $\sim 30$ meq kg-1 per day of bicarbonate were required in early infancy, but during childhood and adolescent years, she was able to maintain her bicarbonate level with doses of $\sim 10-20$ meq kg-1 per day. Although the normal range for bicarbonate depends on age, we labeled $20 \mathrm{mmolI}^{-1}$ with a dotted line for reference. (b) Reticulocyte count was persistently elevated throughout the lifespan and reflects ongoing hemolysis. The dotted line represents the upper limit of normal range (1.5\%). (c) The proband's weight gain (thick line) is plotted. The 10th, 50th and 90th percentile growth curves from the CDC (2000) growth charts are provided (thin lines). (d) The proband's height (thick line) is plotted. The 10th, 50th and 90th percentile growth curves from the CDC (2000) growth charts are provided (thin lines).

Early diagnosis and treatment is thought to correlate with a better long-term outcome. If a neonate presents with hemolytic anemia and metabolic acidosis, it is important to consider GSSD, ${ }^{14}$ as rapidly fatal GSSD in newborns has been described. ${ }^{15}$ Advanced diagnostic techniques such as antenatal diagnosis can be made by measuring 5 -oxoproline in amniotic fluid ${ }^{16,17}$ or a presumptive diagnosis can be made by detecting the elevation of 5-oxoproline in newborn screen blood spots using tandem mass spectrometry. ${ }^{18}$ Our patient's diagnosis was made within a few days of life, based on urine organic acid analysis and thus, treatment with vitamin $\mathrm{C}$ and $\mathrm{E}$ were started early. Njalsson et al. ${ }^{19}$ demonstrated that early initiation of vitamins $\mathrm{C}$ and $\mathrm{E}$ could prevent the moderate phenotype from progressing to severe phenotype. In a study of 41 patients with GSSD, only $1 / 18$ of the severely affected patients were started on vitamin therapy early in life as compared with $6 / 17$ moderately affected patients. With these data and the fact that there is no significant difference between enzymatic activity in the moderate and severe phenotypes, they posit that early initiation of vitamins could prevent or slow down progression of the disease. There were no specific comments on length of follow-up of this cohort however.

Our patient was diagnosed at birth with moderate disease because of her acidosis and hemolytic anemia. However, at the age of 10 years, she developed seizures, a finding which is more consistent with the severe phenotype. Her seizures were very mild and anti-epileptics were eventually discontinued once she was seizure-free for 5 years. We posit that early initiation of vitamins and combination citric acid, potassium citrate and sodium citrate coupled with good compliance explains her relatively mild course. Another possibility is her seizures were unrelated to her underlying GSSD.

Another area of concern for GSSD patients is growth and developmental delay. Our patient had an episode of weight loss and deceleration in head growth in early childhood that was attributed to chronic metabolic acidosis and that improved with increased doses of her medications. Currently, she has short stature $(<5$ th percentile, Z-score -2.1) with appropriate weight and head circumference. Developmentally, our patient experienced minor speech delays early in life that required speech therapy and there were concerns for mild fine motor delay that resolved without intervention. She had some mild difficulties in school and required extra support in the classroom. However, she is currently performing satisfactorily in high school with plans for attending college.

In conclusion, we describe long-term follow-up of a patient with severe GSSD and good outcome. We attribute early recognition of the disease, initiation of appropriate vitamin therapy and acidosis correction along with excellent compliance from our patient for her success to date. We also believe that the use of selenium as an additional antioxidant has contributed to her relatively mild course. Finally, we recommend testing for GSSD in patients with metabolic acidosis or hemolytic anemia in the newborn period as early recognition and initiation of therapy appears to correlate with better outcomes. 


\section{CONFLICT OF INTEREST}

The authors declare no conflict of interest.

\section{ACKNOWLEDGEMENTS}

PSA is supported by the American College of Medical Genetics Foundation/ Genzyme 2014-2015 Fellowship in Biochemical Genetics. LCB is supported by a fellowship from the National Urea Cycle Disorders Foundation, a fellowship from the Urea Cycle Disorders Consortium (UCDC; U54HD061221), which is a part of the National Institutes of Health (NIH) Rare Disease Clinical Research Network (RDCRN), supported through collaboration between the Office of Rare Diseases Research (ORDR), the National Center for Advancing Translational Science (NCATS) and the Eunice Kennedy Shriver National Institute of Child Health and Human Development (NICHD), and NIH K08DK106453.

Author contributions: All authors above made substantial contributions to the conception or design of the work or the acquisition, analysis or interpretation of data for the work and drafting the work or revising it critically for important intellectual content and gave final approval of the version to be published and agreed to be accountable for all aspects of the work in ensuring that questions related to the accuracy or integrity of any part of the work are appropriately investigated and resolved.

1 Meister, A. \& Anderson, M. E. Glutathione. Ann. Rev. Biochem. 52, 711-760 (1983)

2 Schafer, F. Q. \& Buettner, G. R. Redox environment of the cell as viewed through the redox state of the glutathione disulfide/glutathione couple. Free Radic. Biol. Med. 30, 1191-1212 (2001)

3 Larsson, A., Ristoff, E. \& Anderson, M. E. in Metabolic and Molecular Bases of Inherited Disease (eds Scriver C. R., Beaudet A. L., Sly W. S. \& Valle D.) (McGraw-Hill, New York, USA, 2005).

4 Njalsson, R. Glutathione synthetase deficiency. Cell. Mol. Life Sci. 62, 938-1945 (2005).
5 Ristoff, E., Mayatepek, E. \& Larsson, A. Long-term clinical outcome in patients with glutathione synthetase deficiency. J. Pediatr. 139, 79-84 (2001).

6 Ristoff, E., Hebert, C., Njalsson, R., Norgren, S., Rooyackers, O. \& Larsson, A. Glutathione synthetase deficiency: is gamma-glutamylcysteine accumulation a way to cope with oxidative stress in cells with insufficient levels of glutathione?. J. Inherit. Metab. Dis. 25, 577-584 (2002).

7 Speilberg, S. P., Boxer, L. A., Corash, L. M. \& Schutman, J. D. Improved erythrocyte survival with high dose vitamin $\mathrm{E}$ in chronic hemolysing G6PD and glutathione synthetase deficiency. Ann. Intern. Med. 90, 53-54 (1979).

8 Kumar Pejaver \& Watson, A. H. 5-Oxoprolinuria due to glutathione synthetase deficiency. J. Inher. Metab. Dis. 15, 937-938 (1992).

9 Boxer, L. A., Oliver, J. M., Spielberg, S. P., Allen, J. M. \& Schulman, J. D. Protection of granulocytes by vitamin $\mathrm{E}$ in glutathione synthetase deficiency. N. Engl. J. Med. 301, 901-905 (1979).

10 Pejaver, R. K. \& Watson, A. H. High-dose vitamin E therapy in glutathione synthetase deficiency. J. Inherit. Metab. Dis. 17, 749-750 (1994).

11 Anderson, M. E. Glutathione: an overview of biosynthesis and modulation. Chem. Biol. Interact. 111-112, 1-14 (1998).

12 Janaky, R., Varga, V., Hermann, A., Saransaari, P. \& Oja, S. S. Mechanisms of L-cysteine neurotoxicity. Neurochem. Res. 25, 1397-1405 (2000).

13 Tinggi, U. Selenium: Its role as antioxidant in human health. Environ. Health Prev. Med. 13, 102-108 (2008).

14 Ben Ameur, S., Aloulou, H., Nasrallah, F., Kamoun, T., Kaabachi, N. \& Hachicha, M. Hemolytic anemia and metabolic acidosis: think about glutathione synthetase deficiency. Fetal Pediatr. Pathol. 34, 18-20 (2015).

15 Divry, P., Roulaud-Parrot, F., Dorche, C., Zabot, M. T., Contrairie, B., Hagenfeldt, L. et al. 5-Oxoprolinuria (glutathione synthetase deficiency): a case with neonatal presentation and rapid fatal outcome. J. Inherit. Metab. Dis. 14, 341-344 (1991).

16 Erasmus, E., Mienie, L. J., de Vries, W. N., de Wet, W. J., Carlsson, B. \& Larsson, A. Prenatal analysis in two suspected cases of glutathione synthetase deficiency. J. Inherit. Metab. Dis. 16, 837-843 (1993).

17 Manning, N. J., Davies, N. P., Olpin, S. E., Carpenter, K. H., Smith, M. F., Pollitt, R. J. et al. Prenatal diagnosis of glutathione synthase deficiency. Prenat. Diagn. 14, 475-478 (1994)

18 Simon, E., Vogel, M., Fingerhut, R., Ristoff, E., Mayatepek, E. \& Spiekerkotter, U. Diagnosis of glutathione synthetase deficiency in newborn screening. J. Inherit. Metab. Dis. 32, S269-S272 (2009).

19 Njalsson, R., Ristoff, E., Carlsson, K., Winkler, A., Larsson, A. \& Norgren, S. Genotype, enzyme activity, glutathione level and clinical phenotype in patients with glutathione synthetase deficiency. Hum. Genet. 116, 384-389 (2005). 